\title{
Molecular Mobility and Confined Plasticity in NEMS Applications
}

\author{
René M. Overney and Scott Sills \\ Department of Chemical Engineering, University of Washington, Seattle, WA 98195, U.S.A.
}

\begin{abstract}
Many modern and future technological applications involve ultrathin polymer films with a thickness below the 100-nanometer scale, where statistical bulk averaging is jeopardized and interfacial constraints dictate transport properties. In such confined polymeric systems, transport properties strongly depend on molecular relaxation and structural phases that deviate from the bulk. This is particularly relevant in applications involving nano-electromechanical systems (NEMS). In this paper, we address the correspondence between bulk deviating local glass transition values with the non-monotonic plastic deformation properties in ultrathin polystyrene films. Polystyrene serves as a model material in a NEMS application designed to circumvent the superparamagnetic limit associated with magnetic data storage. The application involves data bit writing via an ultrahigh density thermomechanical indentation process. An elaborate frictionvelocity analysis is introduced as a material characterization tool. It provides fundamental insight into the glass forming process, and consequently, the glass transition value in ultrathin spin coated polymer films. The glass transition value is thereby discussed as a phenomenological limit, not unlike an asymptote, to a diverging size of cooperative rearranging regions upon cooling. Unexpected large cooperative clusters up to $40 \mathrm{~nm}$ were observed - a dimension that is noticeable at the 100-nanometer length scale. In the light of MD simulations and their good correspondence with the presented intrinsic friction analysis, the importance of angular and torsional intramolecular motions are particularly emphasized for nanotechnological applications.
\end{abstract}

\section{INTRODUCTION}

Obtaining a fundamental understanding of the glass forming process is crucial in developing novel polymeric materials with unique properties for nanotechnological applications. For instance, thermomechanical terabit-recording[1-3] devised for low powered electronics, and intended to surpass the density restriction imposed by the inherent superparamagnetic limit in digital recording, would greatly benefit from a nanoscopic understanding of the glass forming process in ultrathin interfacially constrained polymer films.

The material property that phenomenologically results from the glass forming process is the glass transition temperature, $\mathrm{T}_{\mathrm{g}}$. The term glass transition is used in the materials community pervasively, implying that it describes a well-understood material phenomena or material property. However, similar to other poorly defined properties, such as friction, a large ambiguity exists. The glass transition is defined as the reversible change in an amorphous material or in amorphous regions of a partially crystalline material, from (or to) a viscous or rubbery condition to (or from) a hard and relatively brittle one. The "midpoint" temperature at which (or the temperature regime over which) the transition occurs is defined as the calorimetric glass transition temperature. A different definition is followed in the course of this text, where $\mathrm{T}_{\mathrm{g}}$ can 
be understood as a phenomenological boundary, not unlike an asymptote, to a diverging size of cooperative rearranging regions upon cooling.

A direct spatial investigation of the glass forming process is, with one exception that is restricted to a narrow temperature range [4], still escaping current instrumental abilities, and this, for both bulk and nanoscale systems. Nanoscale investigations are particularly relevant in small confining systems [5], as in ultrathin films, where interfacial constraints become increasingly important in describing material behaviors.[6-12] The 100-nanometer scale has been shown to have multifarious effects on polymer rheology and relaxation behaviors [13], and consequently on device performances.

\section{EXPERIMENTAL AND INSTRUMENTAL BACKGROUND}

\section{Shear Modulation Force Microscopy SM-FM}

A direct approach to relaxation properties is the measurements of mechanical responses. Particularly for ultrathin films, the SFM based shear modulation force microscopy SM-FM [12] has proven itself to be a very reliable method, in excellent agreement on the macroscale with differential scanning calorimetry and electron spin resonance over a wide range of molecular weight, and applicable to many polymer systems [13-17]. The method, depicted in Figure 1, is briefly described as follows: A nanometer sharp SFM cantilever tip is brought into contact with the sample surface. While a constant load is applied, the probing tip is laterally modulated with a "no-slip" nanometer amplitude, i.e. a small enough amplitude that guarantees no relative probesample slippage. The modulation response is analyzed using a two-channel lock-in amplifier, comparing the response signal to the input signal. The response amplitude is a measure of the contact stiffness, $k_{c}$, described as $k_{c}=8 G^{*} a$ in the fully elastic limit, where $G^{*}$ and $a$ are the reduced shear modulus and the contact area, respectively [15]. By maintaining a stationary tip contact at a constant load, changes in the contact area occur due to creep. The experimental observable in SM-FM, the shear modulation response amplitude reflects the changes in normal and shear modulus of the material. The response amplitude is recorded and plotted versus temperature. Thermally activated transitions in the material, such as the glass transition, are determined from a kink in the response curve, as documented in Figure 1. The uncertainty of the temperature controller $\pm 0.1 \mathrm{~K}$.
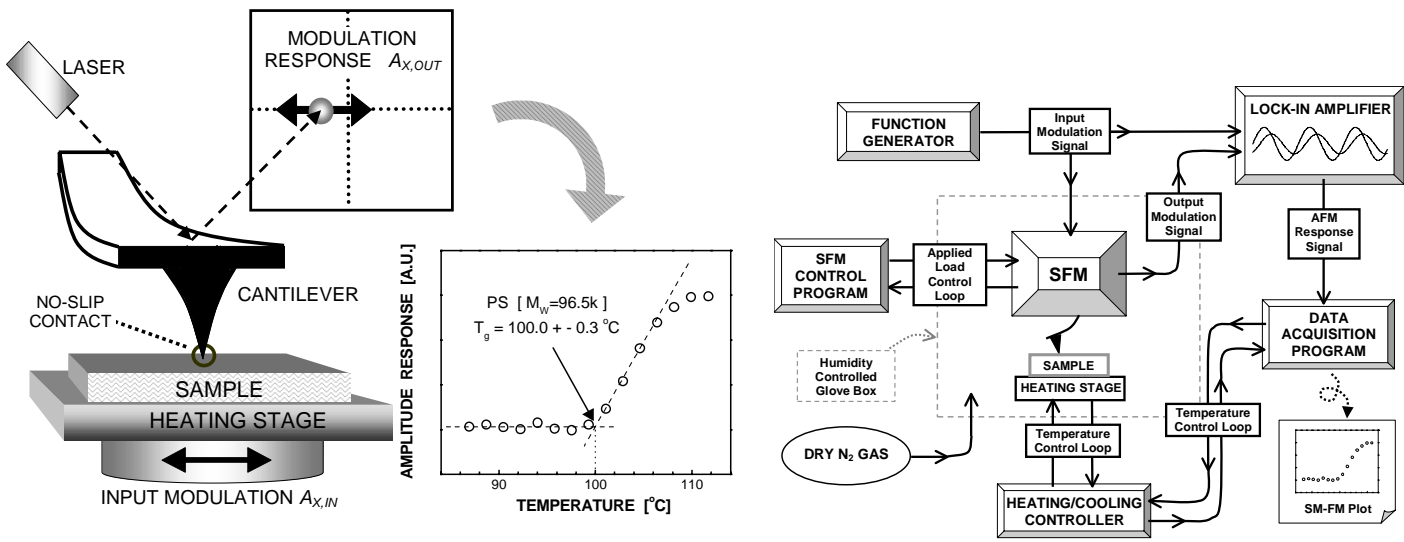

Figure 1: (Left) Schematic of SM-FM. (Inset) Typical SM-SM plot. $\mathrm{T}_{\mathrm{g}}$ is identified by the kink in the plot. (Right) Schematics of the experimental setup. 
The qualitative behavior after a kink upon heating can be either deceasing or increasing (as shown here for PS), and interpreted as follows:

(a) The contact stiffness decreases after the kink, because the material's shear modulus decreased but the contact area has remained the same. This has been observed for monolayers of perfluoropolyethers [18], and for structural reorganizations in complex photonic materials involving chromophores confined in dendron cages [19].

(b) The contact stiffness increases because of an increase in the contact area. This is a more common observation for polymer films, particularly for the glass transition, where the internal pressure drops and the tip sinks in until the pressure is equilibrated. Typically, changes in the contact area dominate changes in the modulus in nano-experiments.

\section{Hot-Tip Scanning Force Microscopy (HT-SFM)}

It is predicted that within a few years, magnetic storage technology will reach the wellknown superparamagnetic limit $\left(\sim 100 \mathrm{~Gb} / \mathrm{in}^{2}\right)$. The approach of this fundamental limit has motivated investigations of completely new data storage techniques, for example, localized mechanical probes or holographic methods [20]. A currently available tool that is simple, yet demonstrates long-term potential, is a nanometer-sharp probe [20]. Such probes are currently used in scanning force and scanning tunneling microscopes (SFM and STM) for sub-micron to sub-nanometer investigations. The potential for using an SFM probe in data storage was first demonstrated by Mamin and Rugar in the early 1990's [21]. Recently, IBM Research Laboratories demonstrated ultra high storage densities of $0.5-1.0 \mathrm{~Tb} / \mathrm{in}^{2}$ with single probe thermomechanical storage, and developed a highly parallel two dimensional array of probes to address the issue of limited single probe data rates [20]. This high areal storage density makes thermomechanical data storage systems very attractive as a potential future technology in handheld applications, offering several gigabyte capacity and low power consumption at megabyte/second rates.

The concept of thermomechanical data-storage relies on the writing, reading, and erasing of nanometer sized data-bit indentations within a suitable polymer storage medium. A local probe, similar to an SFM cantilever tip, we will refer to it as hot-tip SFM (HT-SFM), is employed for the necessary storage operations. The tip is heated resistively, by applying a pulsed bias, $\varepsilon$, between the two legs of the cantilever as sketched in Figure 2.

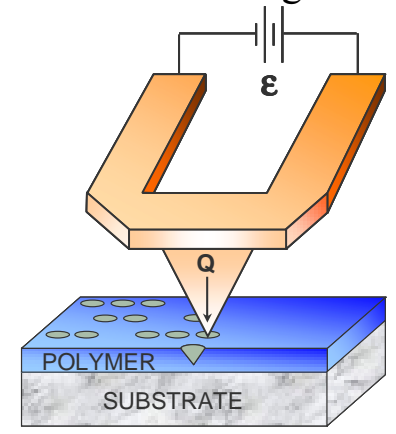

Figure 2: Concept of thermo-mechanical data storage; involving 20-50nm thick polymer film.

The electrostatically applied force is proportional to the square of the absolute difference between the tip and sample biases, and can be determined from force-displacement measurements. The force is obtained by measuring the additional z-piezo displacement required to reach the snap-out instability during a force-displacement curve as a function of the applied 
sample bias. Thereby, the electrostatic force is the product of the additional z-displacement and the normal spring constant of the lever.

Bit indentations are written by a combination of locally softening the polymer, above $T_{g}$, by heating the probe tip, while simultaneously applying a force to the probe, pressing it into the polymer film. Recorded bits are read-back by measuring a change in heat flux (resistance across the heater tip) from the probe to the polymer. Erasing bits requires local heating with the probe to a temperature above $T_{\mathrm{g}}$, where the displaced polymer may backflow into the original indentation. The mechanical and material properties of the polymer storage media are critical to thermomechanical storage operations. The ideal polymer medium should be easily deformable for bit writing; however, the written bits must be stable against wear, thermal degradation, and dewetting, offering a minimum reliability of ten years. The polymer's glass transition temperature $\left(\mathrm{T}_{\mathrm{g}}\right)$ is one of the main parameters determining the threshold writing temperature.

\section{RESULTS AND DISCUSSION}

\section{Critical Length Parameter during the Glass Forming Process}

The following experiments were conducted with a SFM under nitrogen with relative humidity below $\sim 7 \%$ using a bar shaped silicon cantilever (Nanosensors ${ }^{\mathrm{TM}}, k_{N}=0.164 \mathrm{~N} / \mathrm{m}$, $\left.k_{T} \sim 80 \mathrm{~N} / \mathrm{m}\right)$. The normal spring constant for a bar shaped and bare silicon cantilever is rather well determined (error $<10 \%$ ) with $[22,23]$

$$
\begin{aligned}
& k_{N}=\frac{E w t^{3}}{4 l^{2}}, \text { and } \\
& t=6.190415 \sqrt{\frac{\rho}{E}} f_{1} l^{2}
\end{aligned}
$$

where $l, w, t$ are the length, width, thickness of the cantilever, respectively, $E$ is the Young's modulus and $\rho$ the density of silicon. The first resonance frequency in normal direction, $f_{1}$, is measured.

The applied load ranged from $-102 \mathrm{nN}$ to $36 \mathrm{nN}$, and the corresponding normal forces, including adhesion, were between 31 and $373 \mathrm{nN}$. Scanning was performed over a $2.0 \mu \mathrm{m}$ range with a $20 \mathrm{~nm}$ line-spacing to avoid memory effects from previous scans. Isothermal frictionvelocity curves were recorded over a velocity range from $0.01-20 \mu \mathrm{m} / \mathrm{s}$.

A first glance of the impact of the glass transition on the friction coefficient, $\mu$, is provided in Figure 3. Illustrated is a transition in the frictional dissipation mechanism originating from side chain relaxations in the glass phase, Fig. 4(a), and backbone relaxation in the melt phase, 48(b). The transition regime starting at $\sim 373 \mathrm{~K}$ is not abrupt but ranges over $15 \mathrm{~K}$. The glass transition temperature, $\mathrm{T}_{\mathrm{g}}=373 \pm 1 \mathrm{~K}$, determined by SM-FM, was found to correspond with the starting temperature of the transition regime in $\mu(\mathrm{T})$. The origin for the frictional dissipation in Figure 3 was determined from isothermal friction velocity analyses, $\left.\mathrm{F}(\mathrm{v})\right|_{\mathrm{T}}$, below and above the glass transition temperature. Superimposed friction-velocity plots are provided in Figure 4 . The Arrhenius representation of the horizontal shift factor, $\mathrm{a}_{\mathrm{T}}$, provides an activation energy, $\mathrm{E}_{\mathrm{a}}$, of $7 \mathrm{kcal} / \mathrm{mol}$ below T $\mathrm{T}_{\mathrm{g}}$, Fig. 4(a), and $88 \mathrm{kcal} / \mathrm{mol}$ above $\mathrm{T}_{\mathrm{g}}$, Fig. 4(b). The $7 \mathrm{kcal} / \mathrm{mol}$ frictional activation barrier below $\mathrm{T}_{\mathrm{g}}$, was found to correspond to the energy attributed to the side chain phenyl ring rotation in atactic polystyrene [11]. The $88 \mathrm{kcal} / \mathrm{mol}$ activation energy coincides with the $90 \mathrm{kcal} / \mathrm{mol}$ energy barrier for the backbone $\alpha$-relaxation process.[24] 


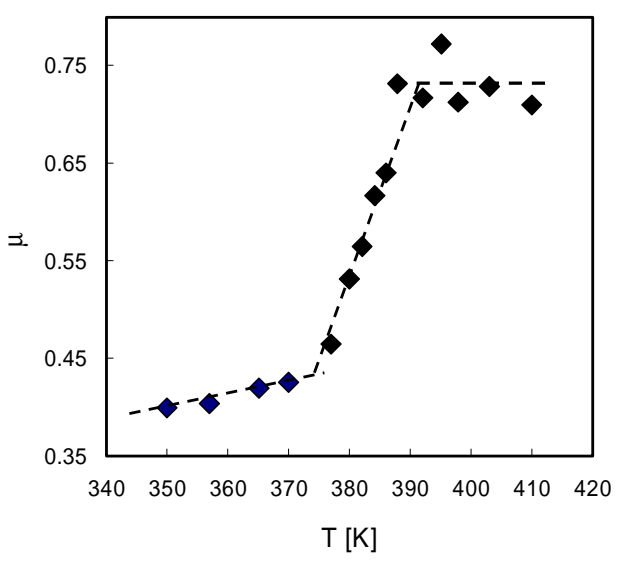

Figure 3: Friction coefficient, $\mu$, around the glass transition of monodisperse $\left(M_{w} / M_{N}=\right.$ $1.04)$ atactic polystyrene $\left(\mathrm{M}_{\mathrm{w}} 96.5 \mathrm{k}\right)$.

The qualitative difference in $\left.\mathrm{F}(\mathrm{v})\right|_{\mathrm{T}}$ curves below and above $\mathrm{T}_{\mathrm{g}}$ is insignificant as a bellshaped friction-velocity behavior can be obtained in both regimes. Under ideal conditions, it is only a question of the velocity range. The bell shape of $\left.\mathrm{F}(\mathrm{v})\right|_{\mathrm{T}}$ originates from the interplay of two dominating time scales: The extrinsic observation time, $\tau_{e}$, dictated by the sliding velocity, $\mathrm{v}$, and the intrinsic material response time, $\tau_{m}$. In the vicinity of the peak, $\mathrm{F}_{\max }$, of the bell curve, the two competing processes occur on comparable time scales. We refer to $F_{\max }$ as the $\alpha$-peak intensity based on the continuity of $\mathrm{a}_{\mathrm{T}}$ above $\mathrm{T}_{\mathrm{g}}$. The friction force increases or decreases with increased sliding velocity depending on whether the extrinsic time trails or leads the material response time, respectively. Generally the interplay between intrinsic and extrinsic times is discussed with their ratio, the Deborah number. No bell-shaped friction-velocity isotherms could be observed below $T_{g}$, because it would demand high sliding speeds of $\mathrm{mm} / \mathrm{s}$ to $\mathrm{cm} / \mathrm{s}$, unachievable with a conventional SFM.

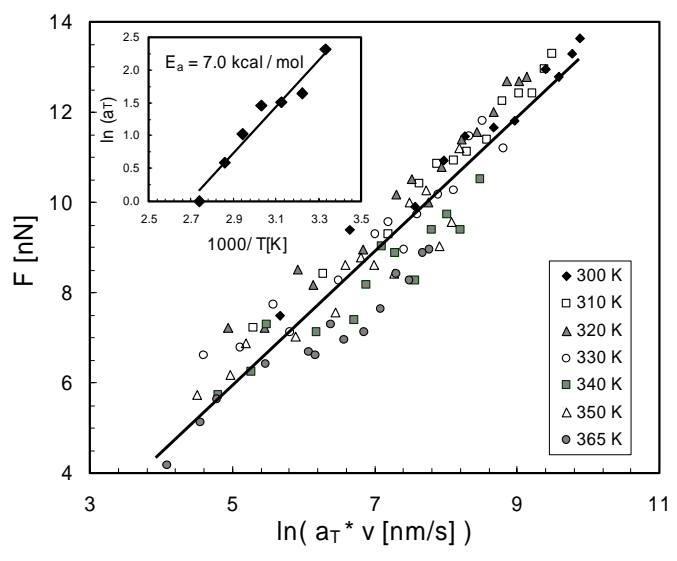

(a)

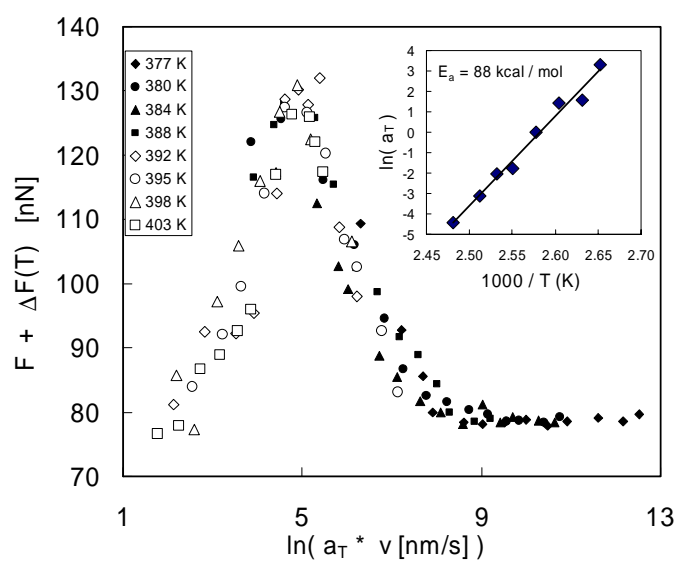

(b)

Figure 4: Superposed $F(v)$ isotherms (a) below $T_{g}[11]$ and (b) above $T_{g}=373 \mathrm{~K}$. (Insets): Arrhenius representations of the horizontal shift factors, $\mathrm{a}_{\mathrm{T}}$, provide activation energies, $\mathrm{E}_{\mathrm{a}}$, of 7 and $88 \mathrm{kcal} / \mathrm{mol}$. 
The location of the $\alpha$-peak intensity $\mathrm{F}_{\max }$ is temperature dependent. A closer look at Figure 4(b) reveals that for lower or higher temperatures (indicated by filled and empty symbols), the $\mathrm{F}(\mathrm{v})$ curves are asymmetrically distributed in regards of $\mathrm{F}_{\max }$. They occur predominantly at velocities above or below $\mathrm{v}_{\max }$ (the velocity corresponding to $\mathrm{F}_{\max }$ ), respectively. At lower temperature values, i.e., from $377-388 \mathrm{~K}$, the material reveals a sluggishness in its response abilities, which results in an increase in the Deborah number, i.e., an increase of the extrinsic time over the intrinsic relaxation time of the material. Thus, an asymmetry of the data distribution in Figure 4(b) in regards to the $\alpha$-peak intensity, as further illustrated in Figure 5(a), has to be anticipated, considering that the experiments were conducted over the same velocity range for all temperatures. It is interesting to note that the temperature dependence of the friction coefficient in Figure 3 also distinguishes the 377-388 K temperature range to be different from the polymer melt. We referred to it as a transition regime from the melt to the glass (or vice versa).

While the superposition-shifts below $\mathrm{T}_{\mathrm{g}}$ only involved the classical horizontal shifts providing the $\mathrm{a}_{\mathrm{T}}$ factors, Fig. 5(a), additional vertical shifts, $\Delta \mathrm{F}$, as illustrated in Figure 5(b), were necessary between $T_{g}$ and $T_{g}+15 \mathrm{~K}$. Inspection of the $\alpha$-peak intensity, $F_{\max }(T)$, in Figure 6 reveals the maturation of the $\alpha$-relaxation from $T_{g}$ to $T_{g}+15 \mathrm{~K}$. The strong $4.8 \mathrm{nN} / \mathrm{K}$ temperature dependence of $\mathrm{F}_{\max }$ in the transition regime is, in essence, caused by the heterogeneity of two structural phases. Conceptually, small domains of the melt phase begin to appear at $\mathrm{T}_{\mathrm{g}}$, yielding a relatively weak $\alpha$-peak intensity. As the temperature increases, remnant glassy domains are consumed by the melt and the $\alpha$-peak intensity increases. Once the melt phase is fully developed at $\mathrm{T}>\mathrm{T}_{\mathrm{g}}+15 \mathrm{~K}$, a temperature independent $\alpha$-peak intensity is observed. This behavior is in accordance with the current understanding of the glass forming process on cooling between the crossover temperature, $T_{c}$ and $T_{g}$, where $T_{c}>T_{g}$. Above $T_{c}$, glass forming polymers show a single peak relaxation process. As the temperature is reduced below $\mathrm{T}_{\mathrm{c}}$, the peak splits into two; a slow ( $\alpha$-backbone) and a fast (side chain) relaxation. [25, 26] As the temperature is further reduced toward $\mathrm{T}_{\mathrm{g}}$, the $\alpha$-peak intensity diminishes and disappears at $\mathrm{T}_{\mathrm{g}}$, while the side chain relaxations continue below $T_{g}$ [25]. In our experiment, only the $\alpha$-peak is observed above $\mathrm{T}_{\mathrm{g}}$ (Fig. 4b). The time-scale for the fast process (side chain) relaxation peak is not directly accessible with the current SFM setup. The existence of structural heterogeneity around $\mathrm{T}_{\mathrm{g}}$ is consistent with conclusions drawn from isothermal multidimensional nuclear magnetic resonance (NMR), dielectric spectroscopy, photobleaching, dynamic light scattering, and quasi-elastic neutron scattering studies [27].
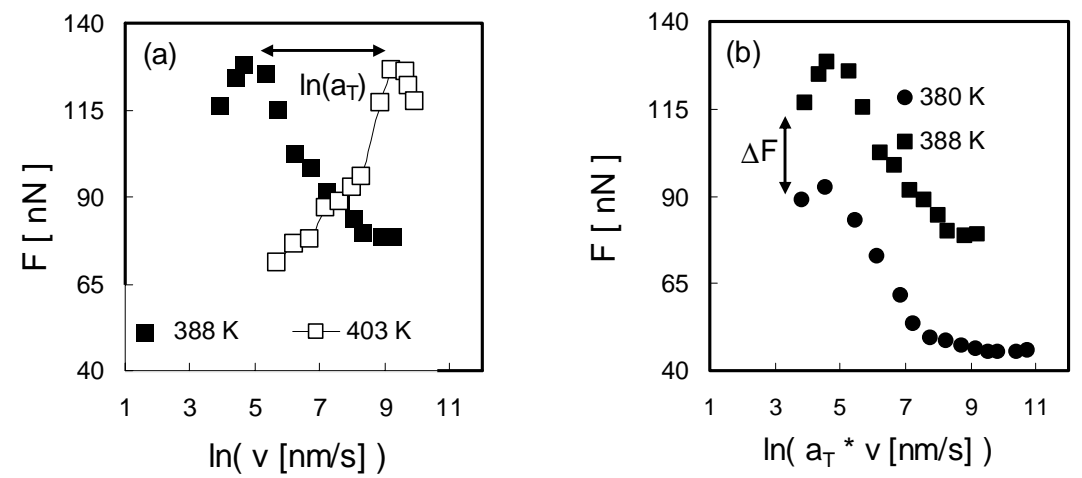

Figure 5: Friction data representative superposition shifts: (a) Horizontal shift applied to all data. (b) Vertical shift only necessary for data between $\mathrm{T}_{\mathrm{g}}$ and $\mathrm{T}_{\mathrm{c}}=388 \mathrm{~K}$. 
The growth regime of the $\alpha$-peak intensity, Fig. 6 , above $T_{g}$ corresponds to the transition regime found in friction coefficient measurements, Fig. 3. It defines a regime of heterogeneous glassy-melt phase in which temporal and spatial cooperation of motions are expected.

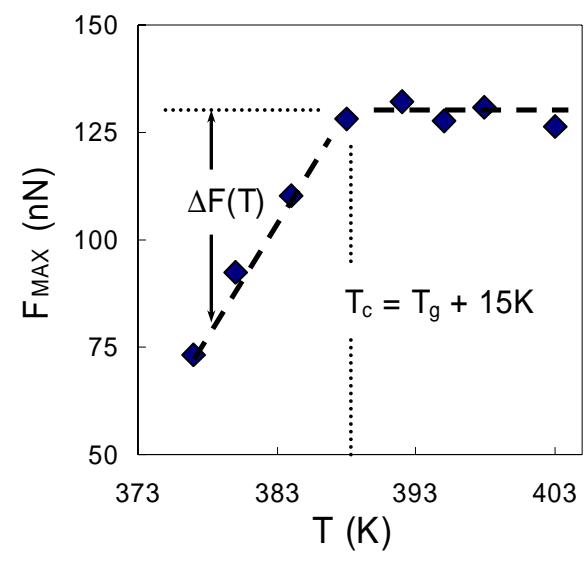

Figure 6: Growth of the $\alpha$-peak intensity $F_{\max }$ above $T_{g}$.

To address the length scale for cooperative motion during the $\alpha$-relaxation, we combined the friction force data with $\alpha$-relaxation time measurements from dielectric spectroscopy[28] for polystyrene of comparable molecular weight $\left(\mathrm{M}_{\mathrm{w}}=90.0 \mathrm{k}, \mathrm{M}_{\mathrm{w}} / \mathrm{M}_{\mathrm{n}}=1.06\right)$. Thereby, the $\mathrm{F}(\mathrm{v})$ peak intensity is analogous to the loss peak in dielectric spectroscopy, where maximum absorption occurs when the electric field frequency, $\tau_{e}^{-1}$, matches the time scale of the segmental motion, $\tau_{\mathrm{m}}$. In contrast to the loss peak, the friction peak is distinguished by a velocity as opposed to a frequency, and thereby coupling a characteristic length scale with a characteristic time scale. Thus, the cooperation length for $\alpha$-relaxations is given as:

$$
\xi_{\alpha}(T)=v_{\max }(T) \star \tau_{\alpha}(T)
$$

where $v_{\max }(T)$ is the velocity corresponding to the $\alpha$-peak intensity of the $F(v)$ curves, and $\tau_{\alpha}(T)$ is the $\alpha$-relaxation time. This is a relationship, which is widely used, and connects friction to rheology [29].

The cooperation lengths $\xi_{\alpha}(\mathrm{T})$ were determined with $\mathrm{v}_{\max }(\mathrm{T})$ and equation (2) so that the resulting $\alpha$-relaxation times are consistent with those from dielectric spectroscopy. These data are fit with a Vogel-Tamann-Fulcher (VTF) equation, shown in the inset of Figure 7, which expresses the typical non-Arrhenius behavior of the $\alpha$-relaxation time. The resulting cooperation lengths $\xi_{\alpha}(\mathrm{T})$ are presented in Figure 7. It is important to note that the determination of the cooperation length $\xi_{\alpha}(\mathrm{T})$, as presented in Figure 7, did not involve any of the many theoretical models generally used to estimate the correlation length [4, 27]. It involved only the matching of the two experimental data sets from the SFM analysis and the dielectric spectroscopy that are intertwined by the simple functional relationship of equation (2).

Figure 7 exhibits a lower limit of the cooperation length in the fully developed melt $(T>400$ $\mathrm{K})$ of $\sim 0.4 \mathrm{~nm}$, which corresponds to the monomer length. On cooling, the cooperation length increases steadily, within a temperature range of $384 \mathrm{~K}$ and $403 \mathrm{~K}$, from 0.4 to $2.1 \mathrm{~nm}$ following 
a power law behavior of the form $\left(\mathrm{T}-\mathrm{T}_{\mathrm{g}}\right)^{-\phi}$, where $\phi$ is $1.86 \pm 0.09$, Fig. 7 . This temperature behavior of $\xi_{\alpha}(\mathrm{T})$ confirms recent theoretical predictions based on molecular dynamics simulations $\left(\phi=\phi_{1}=1.87 \pm 0.15\right)$ for the spatial correlation of segmental displacements above the critical temperature of the mode coupling theory [30].

We find a strong deviation from the above power law behavior with cluster sizes up to $37 \mathrm{~nm}$ below $388 \mathrm{~K}$. This phenomena can be explained with recent MD simulations that incorporate angular and torsional potentials, and suggest that intramolecular interactions contribute to slowing down the dynamics close to $\mathrm{T}_{\mathrm{g}}[31,32]$. The MD simulation predicts an amplified power-law behavior with an exponent $\phi$ of 2.9. A power law fit to $\xi_{\alpha}(\mathrm{T})$ below $388 \mathrm{~K}$ in Figure 7 reveals an exponent $\phi$ of $3.0 \pm 0.2$. Thus, in the light of this MD simulation, $388 \mathrm{~K}$ could be interpreted as a critical temperature below which intramolecular interactions alter the dynamics of the clustering phenomenon.

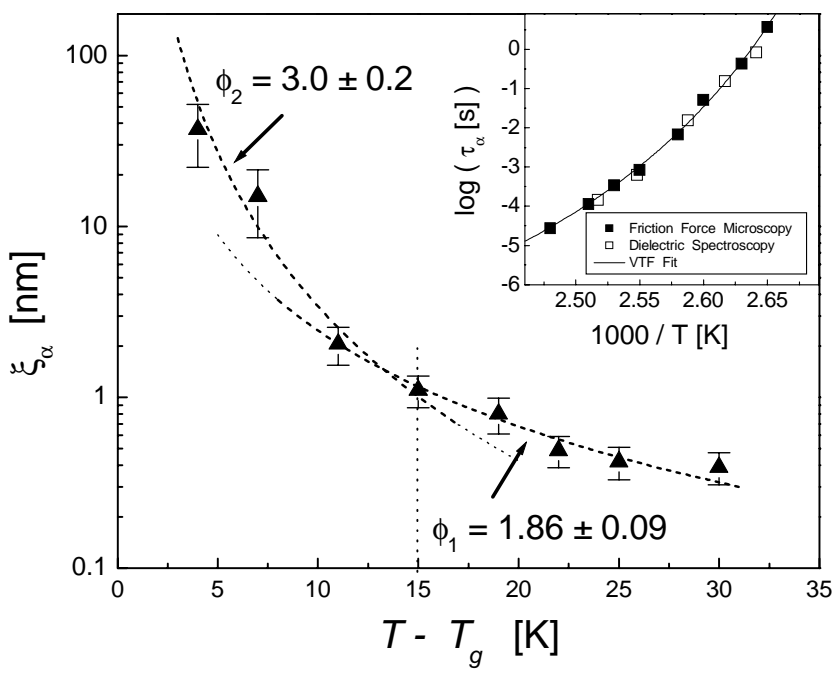

Figure 7: The length scale for cooperative motion during the $\alpha$-relaxation of polystyrene near $\mathrm{T}_{\mathrm{g}}=373 \mathrm{~K}$. The dashed lines show the best fits of the power relation $\xi_{\alpha} \sim\left(\mathrm{T}-\mathrm{T}_{\mathrm{g}}\right)^{-\phi}$ with $\phi_{1}=1.86 \pm 0.09(\mathrm{~T} \geq 384 \mathrm{~K})$ and $\phi_{2}=3.0 \pm 0.2(\mathrm{~T} \leq 388 \mathrm{~K})$. Inset: Polystyrene $\alpha$-relaxation times, $\tau_{\alpha}$, determined from the SFM friction peak compared to dielectric spectroscopy measurements[28]. The solid line represents a fit of the Vogel-Tamman-Fulcher equation, $\tau(\mathrm{T})=\tau_{\mathrm{o}} \exp \left(\mathrm{B} /\left(\mathrm{T}-\mathrm{T}_{\mathrm{o}}\right)\right)\left(\tau_{\mathrm{o}}=2.06^{*} 10^{-12} \mathrm{~s}, \mathrm{~B}=1019 \mathrm{~K}, \mathrm{~T}_{\mathrm{o}}=341 \mathrm{~K}[28].\right)$

The size of the cooperation length near the glass transition temperature can be considered a critical parameter in finite size limited systems such as ultrathin films, and shall be further illuminated in the following discussion.

Based on the results above, the size of the cooperative rearranging regions (CRRs), $\xi_{\alpha}(T)=\left(T-T_{g}\right)^{-\phi}$, grows in bulk PS systems to tens of nanometers close to the calorimetric glass transition value $\mathrm{T}_{\mathrm{g}}$, Fig. 7. Thereby, $\mathrm{T}_{\mathrm{g}}$ can be understood as an asymptote upon cooling to a diverging $\xi_{\alpha}(\mathrm{T})$ as depicted in Figure 8(a) for $\left(\phi_{1}, \phi_{2}\right)$. It is interesting to note from the power law behavior of $\xi_{\alpha}(\mathrm{T})$ that intramolecular interactions impact the growth of the CRRs significantly by an increase of the exponent $\phi$ from $\phi_{1}=1.86 \pm 0.09$ to $\phi_{2}=3.0 \pm 0.2$. For spin coated and thermally annealed ultrathin films, one deals in addition with material that is dimensionally constrained and structurally modified $[11,33]$. Considering these constraints, it can be expected 
that also the power law exponent is affected, as the length scale over which the intramolecular interactions will be modified. For a system of less mobility, the temperature region over which the glass forming process occurs, shrinks as indicated with the exponential growth factor $\phi_{2}{ }^{*}$ and depicted with a dashed line in Figure 8(a). Consequently, the phenomenological limit $\mathrm{T}_{\mathrm{g}}$ would be reached earlier upon cooling, explaining the initial increase in $T_{g}$ as shown in Figure 8(b). For a boundary system of increased mobility, as found for films thinner than $\sim R_{g}$ (radius of gyration), $[11,33]$ the phenomenological limit, $\mathrm{T}_{\mathrm{g}}$, would be reached later upon cooling.

Figure $8(\mathrm{~b})$ is a qualitative representation as found for ultrathin polystyrene films, for thicknesses below $\delta_{\mathrm{o}} \sim 100 \mathrm{~nm}$. More information about the origin for thickness anisotropy in such thin films are found in references $([11,33])$.
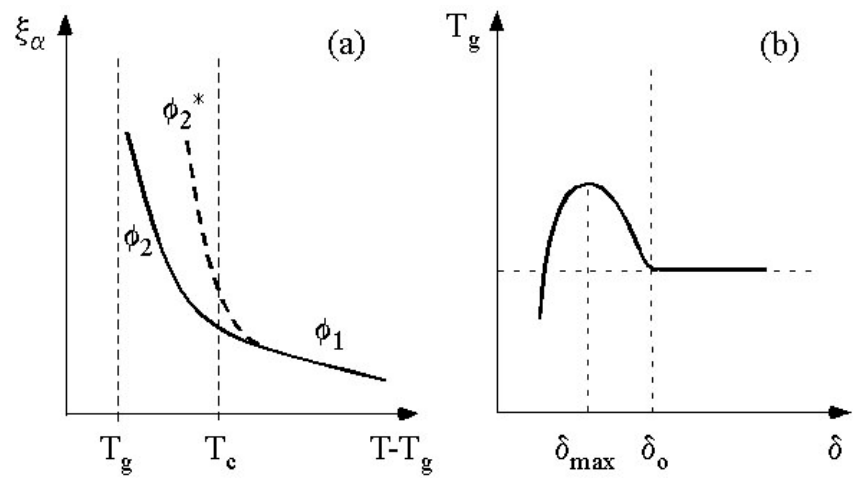

Figure 8: Sketch of the impact of finite size limitations on the dynamics of the glass transition process. (a) Growth behavior of CRRs upon cooling for bulk systems $\left(\phi_{1}\right.$ and $\left.\phi_{2}\right)$ and in spincoated ultrathin confined films $\left(\phi_{1}\right.$ and $\left.\phi_{2}{ }^{*}\right)$ for a film thickness $\delta$ within $\delta_{\max }$ and $\delta_{0}$ as defined in 12(b). (b) Qualitative behavior of the non-monotoneous $T_{\mathrm{g}}$-profile in spin coated ultrathin films of polystyrene.[11, 33]

The impact of constrained phenomenological properties, i.e., the bulk deviating $\mathrm{T}_{\mathrm{g}}$ profile, on a NEMS (NanoElectroMechanical Systems) application, i.e., the thermomechanical bit writing process introduced above, is here discussed. A representative data-bit imprint in thin polystyrene films $\left(\mathrm{M}_{\mathrm{W}}=12 \mathrm{k}, 16-163 \mathrm{~nm}\right.$ thick $)$ with its relevant indentation parameters is presented in Figure 9. The vertical parameters are the indent depth, $d$ and the rim height, $z$, and the horizontal parameters are the indent diameter, $D_{i}$ and the rim diameter, $D_{R}$.

During the indentation of a rigid plastic solid, the displaced material generally appears in the piled-up rim around the periphery of the indentation site; but with elastic-plastic materials, most, if not all, of the displaced material is accommodated by radial expansion of the elastic surroundings, with an imperceptible change in the surface dimensions of the indented material. It is known from indentation and scratch hardness studies on polymeric materials that the measured yield stress is strongly dependent on both the indenter geometry (related to strain, $\tan \beta$ ) and the applied strain rate [34-39]. Pile-up is also influenced by the strain hardening behavior of the material. A large capacity for strain hardening advances the plastic zone further into the material, thus decreasing pile-up adjacent to the indenter [40]. Further, for indentations in compliant films, increased rim heights are observed when elastic strain and plastic flow are constrained, or shielded, by a rigid substrate [41-44]. In the case of rigid films on compliant substrates, the plastic yield of the underlying substrate accommodates an enhanced sink-in of the surface around the periphery of indentation sites [41]. 


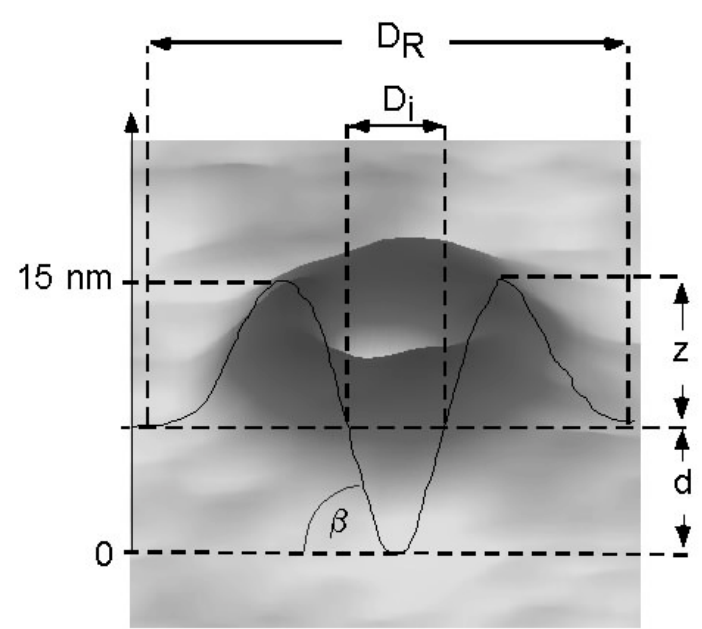

Figure 9: Representative indentation during a $50 \mu$ s pulse at $50-150 \mathrm{nN}$ into a $45 \mathrm{~nm}$ thick PS film. For PS film thicknesses exceeding $\sim 100 \mathrm{~nm}$, the $z / d$ ratio displays a constant value of approximately 0.2 , and reflects the bulk material response. For film thicknesses below $100 \mathrm{~nm}$, the rim height increases with decreasing film thickness.

Examining the ratio of the rim height to the indentation depth, $\mathrm{z} / \mathrm{d}$, provides an assessment of substrate constraints imposed during the indentation process, Figure 10.

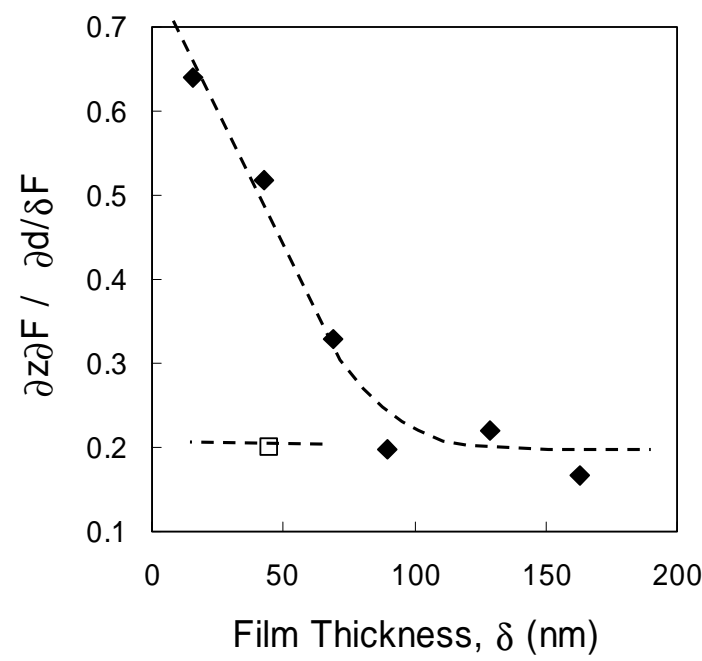

Figure 10: Load $F$ averaged ratio of the rim height and the indentation depth, z/d. Substrate: Bare silicon wafer $(\diamond)$, and silicon wafer coated with a compliant $230 \mathrm{~nm}$ film of polystyrenevinylbenzocyclobutene (PS-BCB) ( $\square$ ).

In Figure 10, the force averaged $z / d$ ratio is reported as a function of film thickness, $\delta$. For a film thickness exceeding $\sim 100 \mathrm{~nm}$, the $\mathrm{z} / \mathrm{d}$ ratio displays a constant value of approximately 0.2 , and reflects the bulk material response. For film thicknesses below $100 \mathrm{~nm}$, the rim height increases with decreasing film thickness. To provide a means of decoupling substrate effects from film thickness effects, one sample (45 nm PS) was prepared with a $230 \mathrm{~nm}$ buffer layer of crosslinked polystyrene-vinylbenzocyclobutene (PS-BCB) between the silicon substrate and the PS film 
Now we consider that the properties of polystyrene are anisotropic below a film thickness of $\sim 100 \mathrm{~nm}$, as discussed above with the non-monotonous $\mathrm{T}_{\mathrm{g}}$-profile. We define an effective modulus as the ratio between the mean pressure $p_{m}=F_{N} / \pi a_{c}{ }^{2}$, where $F_{N}$ is the normal load and $a_{c}$ is the contact radius, and the resulting strain $\tan \beta$. Next we normalize the effective moduli by the constant value found for thick films $(\delta>150 \mathrm{~nm})$, and plot them as a function of the film thickness, Fig. 11. A bell-shaped curve of the normalized effective moduli results that strongly corresponds with the $\mathrm{T}_{\mathrm{g}}$-profile also presented in Figure 11.

Viewing the glass transition as a mobility barrier, an increase in $T_{g}$ offers additional resistance to intermolecular mobility, which intuitively, is accompanied by an increase in the modulus. Hence, the constrained phenomenological transition property, $T_{g}$, strongly impacts the mechanical deformation mechanics, which in the case of thermomechanical recording, can be used to optimize the shape of the indentation. For instance, a restraint over the growth of the indentation rim is achieved by controlling the mobility in the subsurface material. Low rims can be expected for a decreasing modulus gradient in the subsurface material, and vice versa. It has recently been shown that the $T_{g}$-profile, i.e., the temperature location and the magnitude of the $T_{g}$ -maximum can be controlled with the crosslinking density and the molecular weight [45]. As $\mathrm{T}_{\mathrm{g}}$ can be recognized as the asymptote to a diverging $\xi_{\alpha}(\mathrm{T})=\left(\mathrm{T}-\mathrm{T}_{\mathrm{g}}\right)^{-\phi}$, the ultimate fundamental parameter to control the indentation parameters is the growth exponent $\phi(\delta)$.

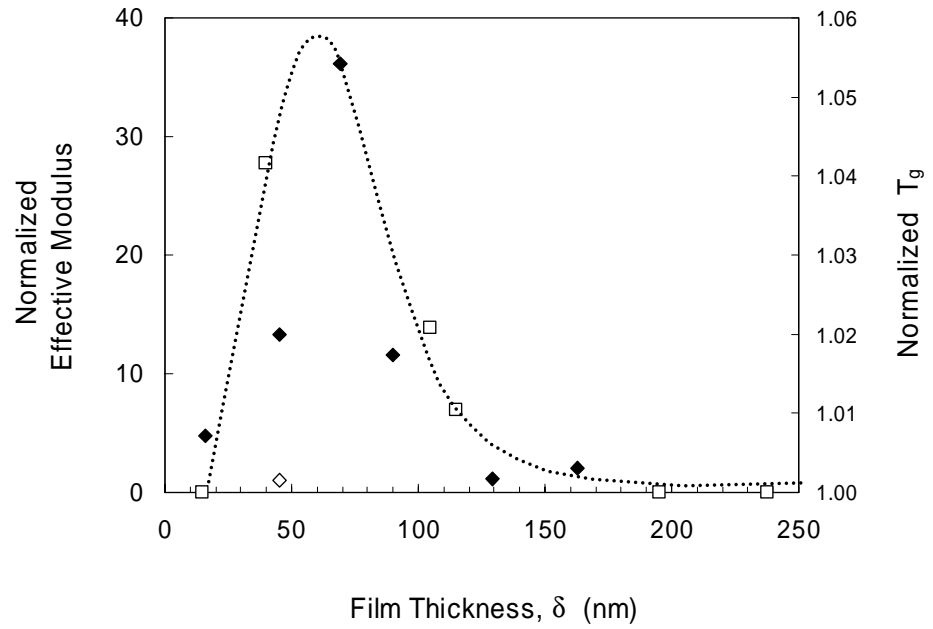

Figure 11: Interfacial thermal ( $\square \mathrm{T}_{\mathrm{g}}$ ) and mechanical response ( $\diamond$ modulus) profiles for thin PS films. The strong correspondence between $\mathrm{T}_{\mathrm{g}}(\delta)$ and the effective modulus underlines the impact of finite size constraints over tens of nanometers in thin spin coated and thermally annealed polymer films. $(\diamond)$ A compliant buffer layer of PS-BCB was placed between PS and the silicon substrate. The $\mathrm{T}_{\mathrm{g}}$ data are adapted from reference ([45]).

\section{SUMMARY}

Control over the heterogeneous dynamics was suggested as a way to influence the $\mathrm{T}_{\mathrm{g}}$ behavior in thin films, and consequently to be used as a material defining engineering tool for nanotechnological applications. Our study on heterogeneous dynamics during the glass forming process benefited from the existence of an $\alpha$-relaxation peak in the friction-velocity isotherms of amorphous glass forming polymers. It provided access to the intrinsic relaxation energy, and 
temperature resolved cooperation lengths by tracking the glass transition process. In the vicinity of $\mathrm{T}_{\mathrm{g}}$, cluster sizes close to $40 \mathrm{~nm}$ were observed - a length scale that was considered to impact the glass transition value for ultrathin polymer films with a thickness on the order of $100 \mathrm{~nm}$ and thinner. The cooperation lengths reported above and their qualitative thermal dependence compare well with predictions based on MD simulations. In the light of MD simulations and their good correspondence with our intrinsic friction analysis, the control of angular and torsional intramolecular motions are imperative in nanotechnological application involving polymers.

The possibility to control critical indentation parameters in thermomechanical recording was addressed. It was suggested that by engineering an appropriate $T_{g}$ profile via "targeted growth" of the cooperative rearranging regions, indentation parameters, such as the indentation rim height, could be appropriately modified. Illustrated was the impact on mobility constraints in thin films of polystyrene. A previously discovered non-monotonuous $T_{g}$ profile over $100 \mathrm{~nm}$ in the substrate boundary films was found to directly influence the effective modulus of the material, and thus, impact the height of indentation rims.

\section{ACKNOLEDGMENTS}

This work was partially supported by IBM, the Petroleum Research Fund administered by the American Chemical Society, the Royalty Research Fund of the University of Washington, and the Nanotechnology Center of the University of Washington.

\section{REFERENCES}

1. U. Durig, G. Cross, M. Despont, et al., Tribology Letters 9, 25 (2000).

2. E. Eleftheriou, T. Antonakopoulos, G. K. Binnig, et al., Ieee Transactions on Magnetics 39, 938 (2003).

3. P. Vettiger, G. Cross, M. Despont, et al., IEEE Transactions on Nanotechnology 1, 39 (2002).

4. H. Sillescu, R. Bohmer, G. Diezemann, et al., Journal of Non-Crystalline Solids 307, 16 (2002).

5. J. M. Drake, J. Klafter, P. E. Levitz, et al., (Materials Research Society, Warrandale, PA, 2001), Vol. 651.

6. Y. Liu, T. P. Russell, M. G. Samant, et al., Macromolecules 30, 7768 (1997).

7. R. M. Overney, D. P. Leta, L. J. Fetters, et al., J. Vac. Sci. Technol. B 14, 1276 (1996).

8. R. M. Overney, D. P. Leta, C. F. Pictroski, et al., Phys. Rev. Lett. 76, 1272 (1996).

9. B. Frank, A. P. Gast, T. P. Russel, et al., Macromolecules 29, 6531 (1996).

10. X. Zheng, M. H. Rafailovich, J. Sokolov, et al., Phys. Rev. Lett. 79, 241 (1997).

11. S. Sills and R. M. Overney, Phys. Rev. Lett. 91, 095501(1 (2003).

12. R. M. Overney, C. Buenviaje, R. Luginbuehl, et al., J. Thermal Anal. and Cal. 59, 205 (2000).

13. J. Frommer and R. M. Overney, in ACS Symposium Series, edited by J. Frommer and R. M. Overney (Oxford Univ. Press, 2001), Vol. 781. 
14. C. Buenviaje, F. Dinelli, and R. M. Overney, in ACS Symposium Series "Interfacital Properties on the Submicron Scale", edited by J. Frommer and R. M. Overney (Oxford University Press, New Orleans, 2000), Vol. 781, p. 76.

15. S. Ge, Y. Pu, W. Zhang, et al., Phys. Rev. Lett. 85, 2340 (2000).

16. F. Dinelli, C. Buenviaje, and R. M. Overney, Thin Solid Films 396, 138 (2001).

17. T. Gray, C. Buenviaje, R. M. Overney, et al., Appl. Phys. Lett. 83, 2563 (2003).

18. R. M. Overney, G. Tyndall, and J. Frommer, in Handbook of Nanotechnolgy, edited by B. Bhushan (Springer Verlag, Heidelberg, 2004).

19. T. Gray, A. K. Y. Jen, and R. M. Overney.

20. P. Vettiger, G. Cross, M. Despont, et al., in Transducers '01. Eurosensors XV. 11th International Conference on Solid State Sensors and Actuators, edited by E. Obermeier (Springer Verlag, Berlin, 2001), Vol. 2, p. 1054.

21. H. J. Mamin and D. Rugar, Appl. Phys. Lett. 61, 1003 (1992).

22. M. Nonnenmacher, J. Greschner, O. Wolter, et al., J. Vac. Sci. Technol. B 9, 1358 (1991).

23. E. Meyer, R. M. Overney, K. Dransfeld, et al., Nanoscience: Friction and Rheology on the Nanometer Scale (World Scientific Publ., Singapore, 1998).

24. R. F. Boyer and S. G. Turley, in Molecular basis of transitions and relaxations, edited by D. J. Meier (Gordon and Breach Science Publishers, Inc., New York, 1978), p. 335.

25. P. G. Debenedetti and F. H. Stillinger, Nature 410, 259 (2001).

26. V. P. Biju, J. Y. Ye, and M. Ishikawa, Journal of Physical Chemistry B 107, 10729 (2003).

27. H. Sillescu, J. Non-Crystalline Solids 243, 81 (1999).

28. C. M. Roland and R. Casalini, J. Chem. Phys. 119, 1838 (2003).

29. K. C. Ludema and D. Tabor, Wear 9, 329 (1966).

30. C. Bennemann, C. Donati, J. Baschnagel, et al., Nature 399, 246 (1999).

31. A. V. Lyulin and M. A. J. Michels, Macromolecules 35, 1463 (2002).

32. A. V. Lyulin, J. J. de Groot, and M. A. J. Michels, Macromol. Symp. 191, 167 (2003).

33. C. Buenviaje, S. Ge, M. Rafailovich, et al., Langmuir, in press. (1999).

34. B. J. Briscoe, P. D. Evans, S. K. Biswas, et al., Trib. Int. 29, 93 (1996).

35. M. J. Adams, A. Allan, B. J. Briscoe, et al., Wear 251, 1579 (2001).

36. C. Ramond-Angélélis, (Ecole Nationale Supérieure des Mines de Paris, 1998).

37. V. D. Jardret and W. C. Oliver, Mat. Res. Soc. Symp. Proc. 594, 251 (2000).

38. T. R. Malow, C. C. Koch, P. Q. Miraglia, et al., Mat. Sci. Eng. A252, 36 (1998).

39. R. Vaisyanathan, M. Dao, G. Ranichandran, et al., Acta Mater. 49, 3781 (2001).

40. J. R. Matthews, Acta Met. 28, 311 (1980).

41. T. Y. Tsui, J. Vlassak, and W. D. Nix, J. Mater. Res. 14, 2204 (1999).

42. T. Y. Tsui and G. M. Pharr, J. Mater. Res. 14, 292 (1999).

43. N. X. Randall, C. Julia-Schmutz, and J. M. Soro, Surface and Coatings Tech. 108-109, 489 (1998).

44. D. E. Kramer, A. A. Volinsky, N. R. Moody, et al., J. Mater. Res. 16, 3150 (2001).

45. S. Sills, J. Frommer, W. Chau, et al., J. Chem. Phys. 120, 5334 (2004). 